\title{
Abordaje quirúrgico y protésico para resolución de defecto Clase IV C de Zucchelli en el maxilar anterior. Reporte de Caso.
}

\section{Surgical and prosthetic approach for resolution of Zucchelli Class IV C defect in the anterior maxilla. Case report.}

\author{
Fernández Cristian ${ }^{1 *}$, Basualdo Javier $^{2}$
}

\begin{abstract}
1. Ayudante, Programa de Especialización en Rehabilitación Oral. Escuela de Graduados, Facultad de Odontología, Universidad de Chile, Santiago, Chile.

2. Profesor Asistente, Programa de Especialización en Implantología Buco Máxilo Facial. Escuela de Graduados, Facultad de Odontología, Universidad de Chile, Santiago, Chile.
\end{abstract}

* Correspondencia Autor: Cristian Fernández Rivera| Dirección: Av. Santa María 0596. Providencia, Santiago, Chile. | Teléfono: +56979802017| E-mail: cfernandez@odontologia. uchile.cl

Trabajo recibido el 03/05/2020

Trabajo revisado 18/08/2020

Aprobado para su publicación el 23/08/2020

\section{RESUMEN}

Se describe el caso clínico de una paciente de 37 años, sexo femenino, sana, con sonrisa gingival, la cual presentaba problemas estéticos en relación, a un implante en la 1.1, instalado hace 7 años, el implante no presentaba una correcta posición tridimensional, correspondiendo a una Clase IVc de Zucchelli. El presente reporte clínico describe los pasos quirúrgicos y protésicos para resolver la estética del maxilar anterior pasando de un PES/WES inicial de 8 a un valor final de 16

PALABRAS CLAVE:

Regeneración ósea; Extracción de implante; Estética.

Int. J. Inter. Dent Vol. 14(3); 274-277, 2021.

\section{ABSTRACT}

We describe the clinical case of a 37-year-old female patient, healthy, with a gingival smile, who had aesthetic problems in relation to an implant in 1.1, installed 7 years ago. The implant did not present a correct three-dimensional position, corresponding to a Class IVc according to Zucchelli. This clinical report describes the surgical and prosthetic steps to resolve the aesthetics of the anterior maxilla, going from an initial PES / WES of 8 to a final value of 16 .

KEY WORDS:

Bone regeneration; Implant removal; Esthetic.

Int. J. Inter. Dent Vol. 14(3); 274-277, 2021.

\section{INTRODUCCIÓN}

La evolución de la implantología como especialidad ha ido en constante avance. Las tasas de éxito y supervivencia de los implantes han demostrado que la oseointegración es una realidad indiscutible ${ }^{(1)}$. Sin embargo, el éxito en la terapia con implantes no debe depender únicamente de su supervivencia a largo plazo, sino también de la estabilidad funcional, estética, de tejidos duros y blandos ${ }^{(2)}$

En el sector anterior es fundamental tener en consideración que después de una extracción, diversos procesos de reabsorción se producen a causa de cambios en el hueso alveolar. Anatómica e histológicamente, el hueso alveolar corresponde a una estructura dependiente del diente, que se desarrolla en conjunto con la erupción dentaria. En la primera fase de remodelación la pared lingual y principalmente la pared vestibular de un alveolo post exodoncia sufren una pérdida ósea, a causa de la pérdida del "bundle bone", el cual es nutrido principalmente por el ligamento periodontal. Como consecuencia se produce una pérdida ósea, tanto en sentido vertical como horizontal ${ }^{(3,4)}$. De este modo la inserción de un implante en el sector estético anterior debe ser tomado con precaución. Además, se debe respetar un posicionamiento tridimensional ideal, para evitar que se produzcan defectos estéticos ${ }^{(5)}$. Es fundamental crear las condiciones favorables para la inserción de un implante, no solo proporcionando una cantidad suficiente de tejido duro, sino que también de tejido blando estético y funcional para poder instalar un implante en forma segura. Sin embargo, la prevalencia de defectos estéticos vestibulares alrededor de un implante, no son tan poco comunes, de hecho, algunos autores han reportado hasta un $57 \%$ de incidencia ${ }^{(7)}$

No lograr un buen resultado estético y funcional con rehabilitaciones implantoasistidas podría dar lugar a situaciones poco estéticas que necesitarían otros procedimientos quirúrgicos y restauradores para corregir el defecto estético(5). Recientemente Zucchelli y cols.(2) publicaron una clasificación para dehiscencias/deficiencias vestibulares en relación, a implantes unitarios en la zona estética, no afectados por enfermedad peri-implantaria, con el intento de sugerir un proceso de toma de decisiones para seleccionar el protocolo de tratamiento más apropiado para lograr un resultado estético ideal. La clasificación de las dehicencias/deficiencias vestibulares de la mucosa peri-implante utiliza 2 parámetros a considerar que son: la ubicación vestibulo - palatina de la plataforma del implante que determina las Clase I, Clase II, Clase III y Clase IV; y la dimensión de las papilas mesial y distal respecto a la posición ideal del margen mucoso de la corona soportada por el implante, que establece la subdivisión de las Clases en las subcategorías a, b y c. La clase IV corresponde a la ubicación apical del margen mucoso periimplante respecto al margen gingival del diente natural homólogo, donde la plataforma del implante, evaluada mediante la extracción de la corona, está por vestibular y fuera de la línea recta imaginaria que conecta el perfil de los dientes adyacentes al nivel del margen gingival. En cuanto a la subdivision c, establece que la altura de al menos una papila está al mismo nivel o más apical que la posición ideal del margen de tejido blando de la rehabilitación implantoasistida.

El propósito de este trabajo es mostrar la resolución de un caso clínico de un implante unitario del sector anterior, clasificación IVc de Zucchelli y cols. ${ }^{(2)}$, al cual se le realiza la explantación, debido a su incorrecta posición 3D, además de realizar una regeneración de tejidos duros y blandos para la posterior inserción de un implante mediante cirugía guiada y la rehabilitación final de todo el sector anterosuperior.

\section{INFORME DEL CASO}

Paciente sexo femenino, 37 años, ASA I, consulta por disconformidad 
estética en el tamaño cervico-incisal de diente 1.1 rehabilitado con prótesis fija cementada sobre implante hace 7 años. Las expectativas de la paciente eran altas, con una exigencia estética importante respecto al resultado del tratamiento. En el análisis extraoral se observa una línea de sonrisa alta, la cual expone más de $2 \mathrm{~mm}$ apical del límite amelocementario, correspondiendo a una sonrisa gingival, lo que evidenciaba claramente una desarmonía estética del sector anterosuperior, debido a la alteración del balance gingival, presentando un desafío enorme a la resolución de caso. Para la valoración intraoral se utilizaron criterios estéticos objetivos que permitieran realizar una comparación estandarizada con el resultado del tratamiento a través del Pink and White Esthetic Scores (PES/WES Inicial: 8)(9), encontrándose que las principales dificultades estéticas se relacionaban con la mucosa peri-implantaria: pérdida de papila mesial, distal, alteración del nivel del margen y color (Figura 1).

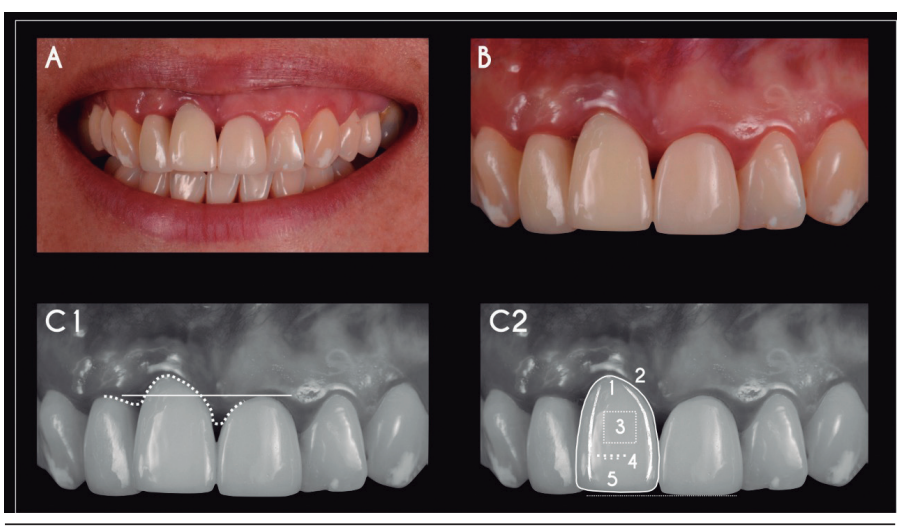

Figura 1. A. Sonrisa máxima. Se presenta una sonrisa gingival con exposición de una banda de encía de mas de $2 \mathrm{~mm}$. B. Visión sector anterosuperior. Se observa un fenotipo gingival fino, alteración del balance gingival y del festoneado marginal en zona de dientes 1.2 y 1.1 respecto a dientes homologos. C1. Indice PES/WES. Se asignan valores de 1 a los parámetros de papila mesial, distal y curvatura de la mucosa facial; asignación de 0 para nivel de la mucosa facial y convexidad-color-textura. Valoración del Pink Esthetic Scores inicial de 3. C2. Indice PES/WES. Se asignan un valor de 2 a textura dentaria, de 1 a forma, color y translucidez, de 0 a volumen/contorno dentario. Valoración del White Esthetic Scores inicial de 5 .

\section{1era Fase Quirúrgica: Injerto de tejido conectivo.}

Se decide retirar la prótesis fija cementada sobre implante, 1 mes antes de la cirugía, con el objetivo diagnóstico de poder evaluar clínicamente la posición tridimensional del implante, observándose una alteración en la angulación y el posicionamiento vestíbulo-palatino de la plataforma del implante (Figura 2), correspondiendo a una Clase IVc, según Zucchelli y cols. ${ }^{(2)}$

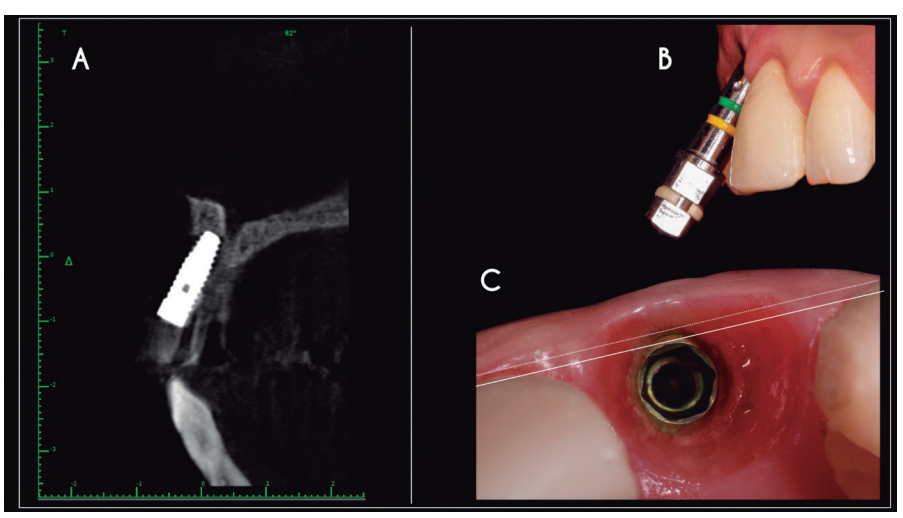

Figura 2. A. CBCT inicial. Implante oseointegrado con ausencia de tabla vestibular. B. Inclinación vestibulo palatina de implante oseintegrado en zona de diente 1.1. Se observa que el eje de inclinación se encuentra fuera del contorno coronario de su homologo. C. Plataforma del implante se encuentra fuera de la linea imaginaria recta que conecta el perfil de los dientes adyacentes al nivel del margen gingival, esto se conoce como Clase IV de Zucchelli y cols.
En primera instancia se decide realizar un abordaje conservador para la corrección del defecto con un injerto de tejido conectivo subepitelial de paladar. Para la obtención del injerto se realiza la técnica de incisión única, según Hürzeler y Weng ${ }^{(10)}$, para luego en el sitio receptor realizar un colgajo de espesor parcial con 2 descargas verticales a la altura de los incisivos laterales para posicionar el injerto conectivo y luego se realiza un desplazamiento coronal del colgajo como describe Zucchelli y cols. en su técnica bilaminar ${ }^{(12)}$. Desde el punto de vista protésico se modificó el diseño del provisional atornillado reduciéndolo en la zona critica y subcrítica para otorgar un espacio adecuado al injerto. En la evaluación a los 3 meses se observa una ganancia de volumen horizontal del tejido peri-implantario, sin obtener una corrección vertical del margen periimplantario respecto al diente homólogo (Figura 3), por lo que se decide como opción terapéutica la explantación atraumática y acondicionamiento de los tejidos duros y blandos para realizar la inserción diferida del implante. No obstante, esta primera cirugía mejoró el fenotipo de la zona, aumentando el espesor mucoso en vestibular.

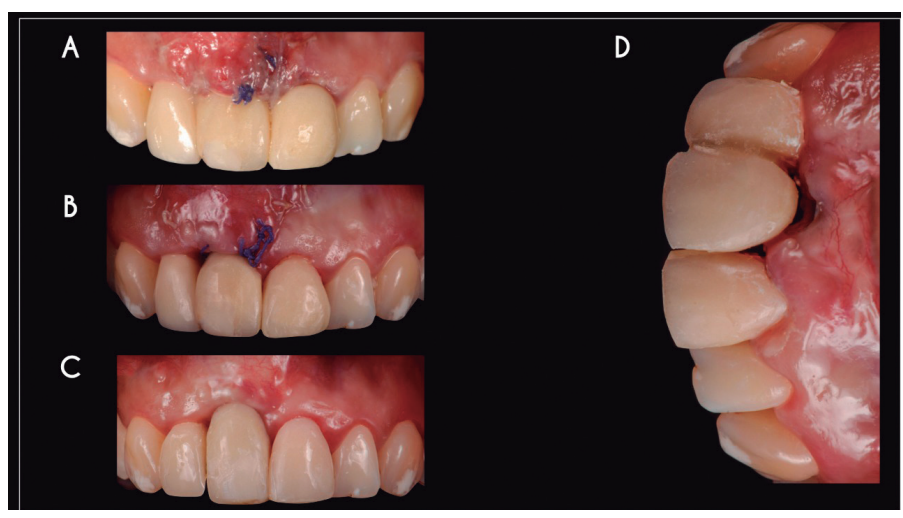

Figura 3. Seguimiento 1era fase quirúrgica $\mathrm{A}$. Control 2 días post injerto conectivo. B. Control 14 días post injerto conectivo. C. Control 3 meses post injerto conectivo. D. Implante sumergido, preoperatorio previo a explantación.

\section{duros.}

2da Fase Quirúrgica: Explantación y reconstrucción de tejidos

Se realizó la explantación atraumática a contratorque del implante Biohorizons $^{\circledR} 4.6$ x $12 \mathrm{~mm}$ y la regeneración simultánea de tejidos duros con la técnica de "Ice Cream Cone"(9) utilizando los siguientes biomateriales: Bio-Oss ${ }^{\circledR}$, Bio-Gide ${ }^{\circledR}$ y Mucograft ${ }^{\circledR}$ Seal como se observa en la figura 4. La paciente fue premedicada con amoxicilina $875 \mathrm{mg}(1$ comprimido cada 12 horas por 7 días, iniciando el tratamiento el día de la cirugía), ketoprofeno $100 \mathrm{mg}$ (1 comprimido cada 12 horas por 3 días inmediatamente después de la cirugía), paracetamol $1 \mathrm{gr}$ (1 comprimido cada 12 horas por 3 días inmediatamente después de la cirugía).

Se realizó el retiro de la prótesis fija unitaria del diente 1.2 para que permitiera una provisionalización fija en cantilever del diente 1.1 Luego de 6 meses se realizó una tomografía computarizada cone beam de control para evaluar el resultado de la reconstrucción de tejidos duros y planificar la inserción del implante, observándose hueso suficiente para lograr estabilidad primaria y un correcto posicionamiento tridimensional del implante (Figura 5a).

Se realizó una planificación digital para cirugía guiada en software coDiagnosti ${ }^{\circledR}$ para inserción de implante Neodent Grand Morse de $3.5 \times 13 \mathrm{~mm}$. Se materializa guía quirúrgica estricta dentosoportada en impresora 3D con tecnología DLP (MoonRay Desktop). Se prueba eje de inserción, ajuste y asentamiento de la guía. Se realiza una incisión supracrestal lineal y semicrevicular (incluyendo los dientes adyacentes), se levantó un colgajo de espesor total para observar clínicamente la osteotomía de la secuencia de fresado. Se realizó la inserción del implante con un torque de inserción de $45 \mathrm{Ncm}$. Se observó un defecto horizontal en la tabla vestibular por lo cual se realizó un relleno con xenoinjerto particulado Bio-Oss ${ }^{\circledR}$. Luego, se realizó una provisionalización inmediata sobre un pilar temporal de Peek (Muñon Pro Peek $\mathrm{GM}^{\circledR}$ ), con un torque de $20 \mathrm{Ncm}$ (Figura 5b,c,d,e,f)

Fase Protésica: Manejo de tejidos peri-implantarios y rehabilitación definitiva.

A las 12 semanas de instalado el implante se realizó un manejo protésico de perfil de emergencia de implante con la técnica de compresión dinámica ${ }^{(11)}$, además de preparaciones biológicas periféricas para carilla en diente 2.2 y de prótesis fija en dientes 1.2 y 2.1 con la 


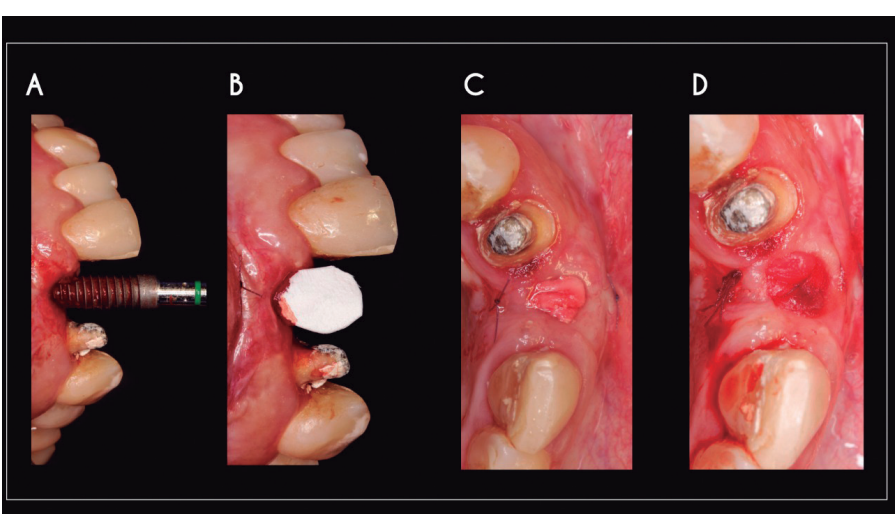

Figura 4. Extracción atraumática de Implante. A. Extracción a contratorque de Implante Biohorizons. B. Sutura de membrana Bio-gide ${ }^{\circledR}$ para técnica Ice Cream Cone. C. Vista oclusal de cierre de membrana Bio-gide ${ }^{\circledR}$. D. Vista oclusal de Mucograt seal ${ }^{\circledR}$ para cierre de regeneración ósea guiada.

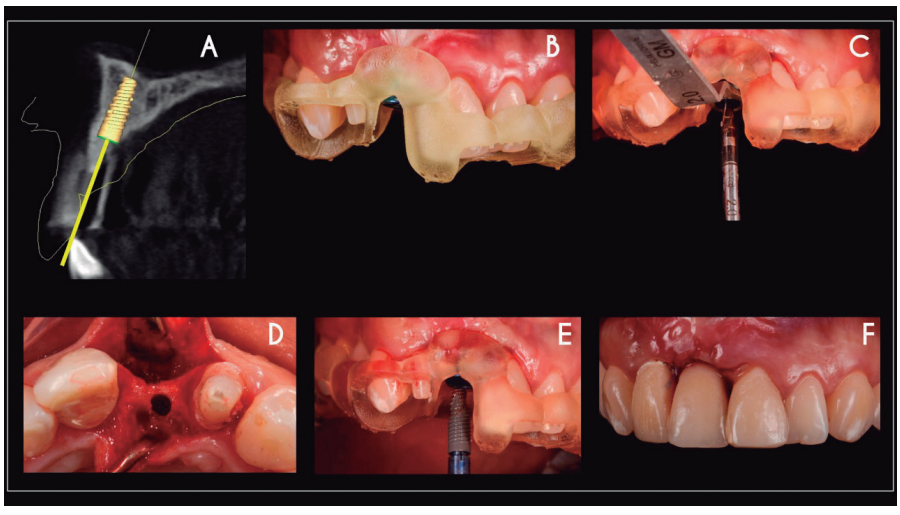

Figura 5. A. Planificación de cirugía guiada en software coDiagnostix®. Se observa una cantidad adecuada de tejido óseo postregeneración. B. Prueba de ajuste. asentamiento y retención de guia quirúrgica estricta dentosoportada. C. Secuencia de fresado guiada. D. Vista oclusal de osteotomía guiada finalizada. E. Instalación de implante Neodent Helix GM $3.5 \times 13 \mathrm{~mm}$. F. Provisionalización Inmediata.

finalidad de poder distribuir adecuadamente los espacios mesiodistal para corregir el apiñamiento en la zona de diente 1.2 y 1.1. La rehabilitación dentosoportada de dientes 1.2, 2.1 y 2.2 se confeccionó en disilicato de litio (E-max Press ${ }^{\circledR}$ ). Para la rehabilitación sobre implante se seleccionó un pilar base de titanio, sobre el cual se realizó un núcleo de zirconia estratificado con cerámica feldespática es vestibular, generando una rehabilitación cemento-atornillada (Figura 6). La cementación se realizó con cemento de resina NX3 Nexus ${ }^{\circledR}$ de Kerr (Figura 7).

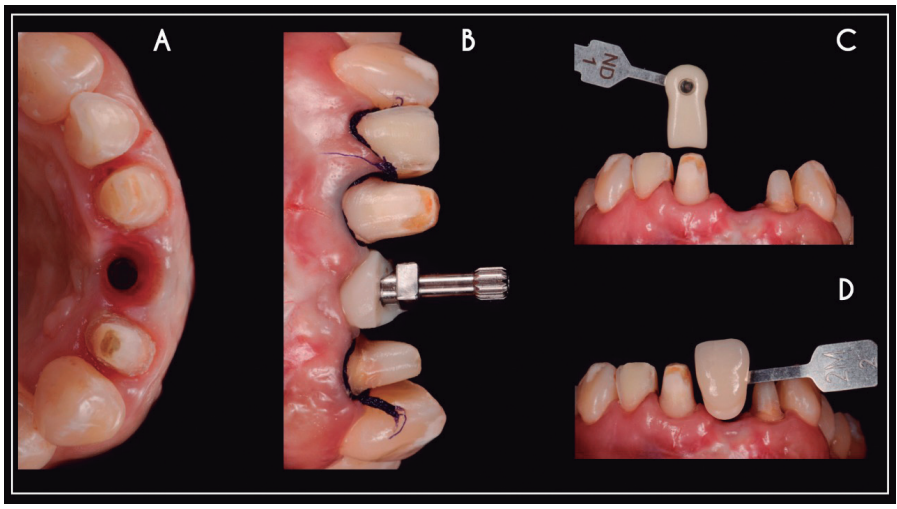

Figura 6. A. Vista oclusal de preparaciones perifericas para PFU en dientes 1.2, 2.1, del trabajo protésico de conformación del perfil de emergencia en el 1.1 y preparación de carilla en diente 2.2. B. Vista frontal previo a impresión de trabajo con personalización de transfer de impresión a cubeta abierta y utilización de técnica de doble hilo para preparaciones biológicas. C. Toma de color del sustrato dentario con guía de color IPS Natural $\mathrm{Die}^{\circledR}$. D. Toma de color final de restauraciones con muestrario Vita 3D master ${ }^{\circledR}$. Se selecciona color $2 \mathrm{M} 2$.

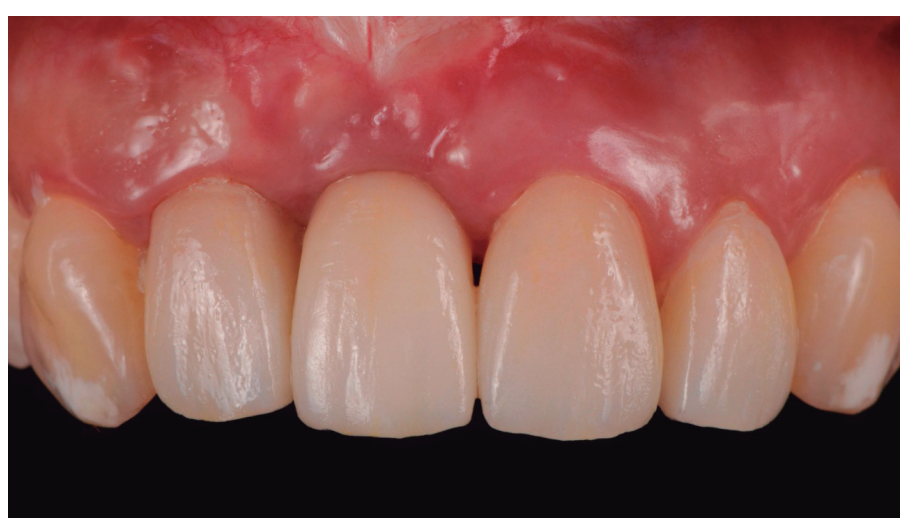

Figura 7. Control rehabilitación sector anterosuperior, 6 meses de seguimiento.

\section{DISCUSIÓN}

Ávila Ortiz y cols. ${ }^{(13)}$ definieron recientemente la importancia del fenotipo gingival como una característica importante para la estabilidad y pronóstico a largo plazo de las rehabilitaciones sobre implantes. En este sentido se han planteado valores umbrales óptimos de los 4 componentes del fenotipo peri-implante que el clínico debe diagnosticar con precisión e intentar mejorar sus características cuando las condiciones dimensionales y morfológicas no sean adecuadas: 1) Ancho de la mucosa queratinizada adecuada $\geq 2 \mathrm{~mm}, 2$ ) Grosor de la mucosa $\geq 2 \mathrm{~mm}$, 3) Altura del tejido supracrestal $\geq 3 \mathrm{~mm}$ y 4 ) Grosor óseo peri-implante $\geq 2 \mathrm{~mm}^{(12)}$

En el presente reporte de caso se observa una ausencia tabla ósea vestibular, asociada principalmente al ancho y a la inclinación en sentido vestibular del implante, lo que se tradujo en una traslucidez del metal a través de la mucosa en relación al implante, además de una pérdida de altura en el zenit vestibular, creando una discrepancia estética en relación a su homólogo.

La literatura refiere como uno de los factores etiológicos mas transcendentes para las deficiencias de tejidos duros y blandos en la terapia de implantes al mal posicionamiento vestibular y el diámetro de los implantes utilizados en el área estética, alcanzando 17.2 veces de que ocurra una alteración de los tejidos periimplantarios y un $14 \%$ de causa de explantación ${ }^{(2,5,6)}$

La cobertura de dehiscencias/deficiencias de tejidos blandos periimplante es un tema relativamente nuevo en la literatura y la mayoría de los estudios son informes de casos o series de casos, por lo que la evidencia disponible es limitada sobre qué enfoque de tratamiento es el más efectivo y predecible ${ }^{(2,6)}$. Las alternativas terapeuticas, de manera general, podrían dividirse en dos categorías: quirúrgicas; y una combinación quirúgicas-protésicas ${ }^{(6)}$. Debido a que la reciente clasificación de dehiscencias/deficiencias peri-implante presentada por Zucchelli y cols. ${ }^{(2)}$ aún no había sido publicada al momento del desarrollo de este caso clínico es que se decidió, como primera alternativa, el abordaje bilaminar quirúrgico-protésico propuesto por el mismo autor el año 2013, ya que reportaba los mejores resultados en cuanto a porcentajes de cobertura de dehicencias ${ }^{(6)}$, respondiendo al objetivo terapeutico principal que era reposicionar el margen mucoso peri-implante al mismo nivel de su homólogo natural.

Zucchelli y cols. con su técnica bilaminar ${ }^{(12)}$ reportaron un $75 \%$ de cobertura completa de las dehiscencias vestibulares en implantes unitarios en la zona estética, en comparación con el diente contralateral homólogo, pero no especificaron el grado de alteración del posicionamiento 3D del implante en sentido vestíbulo-palatino que sin duda tiene un factor pronóstico esencial respecto al rendimiento de la cobertura en la dehiscencia como queda claramente establecido en una nueva publicación de Zucchelli y cols. ${ }^{(2)}$, en donde clasificaron los defectos en 4 categorías con sus respectivas propuestas terapéuticas quirúrgicas. La clasificación IVc establece la condición más crítica, desfavorable y poco predecible, donde la plataforma del implante esta en una posición más vestibular que la línea imaginaria recta que conecta el perfil de los dientes adyacentes al nivel del margen gingival, además de que la altura de al menos una papila, está al mismo nivel o más apical de la posición ideal del margen de tejido blando de la corona soportada por el implante. Zucchelli y cols. ${ }^{(2)}$ proponen para esta condición la remoción del implante, y como quedó demostrado en este informe de caso, no fue posible compensar la deficiencia vertical con un abordaje mas conservador como proponía con su técnica bilaminar, logrando solamente un mejoramiento en el grosor de la mucosa peri-implante. Interesante es poder cuantificar cuanto es la cantidad que se puede ganar en el cubrimiento de una 
dehiscencia o defecto vestibular con una técnica de injerto conectivo, y la revisión sistemática de Rotundo y cols. ${ }^{(14)}$ nos señala que sólo es posible obtener en promedio $1.65 \mathrm{~mm}(1.4-1.8 \mathrm{~mm})$ de cubrimiento de dehiscencias peri-implante al año de tratamiento, situación fundamental de considerar ya que en recesiones mayores a $2 \mathrm{~mm}$ como en este reporte de caso $(2.5 \mathrm{~mm})$, la técnica fue francamente insuficiente como quedo demostrado; esto sin considerar la maduración de los tejidos y el fenómeno de "creeping attachment" que puede mejorar las condiciones estéticas de los tejidos peri-implantarios en el tiempo.

Además cabe señalar que la elección de las técnicas quirúrgicas deben seleccionarse con precaución, ponderando el tipo de defecto/ dehiscencia, eficacia, complejidad, habilidad del operador y sin lugar a dudas los resultados cualitativos (integración estética del injerto), evitando, en lo posible, seleccionar técnicas en el área estética que impliquen descargas o planificando adecuadamente su ubicación, debido a que en la actualidad la manera objetiva de medir los resultados es a través de índices estéticos como el PES ${ }^{(15)}$ o PES/WES ${ }^{(8)}$ que toman en consideración las características cualitativas del fenotipo mucoso periimplantario y/o de la estética blanca de las rehabilitaciones cerámicas. En este informe de caso se utilizó el índice PES/WES para valorar los criterios de éxito de estética rosa y estética blanca, al inicio y al final de tratamiento dando como resultado un PES/WES inicio: 8 y PES/WES final: 16 , superando el umbral de aceptabilidad clínica de 12, lo que revela objetivamente el éxito terapéutico del camino abordado. En concordancia con la apreciación de los autores Belser y cols. ${ }^{(8)}$ respecto al índice PES que utiliza siete variables independientes con la misma importancia cada una de ellas, donde los parámetros 5,6 y 7 que abordan el color del tejido blando periimplantario, la textura y la convexidad facial, no pueden ser considerados con el mismo peso cuando se valora el rendimiento estético de un implante en el sector anterior, respecto a la real trascendencia estética que implica la presencia o alteración de las papilas y el margen del tejido mucoso vestibular. Esta situación se encuentra bien ponderada en el índice PES/WES que, a juicio nuestro, representa de mejor manera la valoración de los distintos parámetros.

Otra alternativa de tratamiento, altamente sustentada en la literatura, es la prótesis fija plural, esta alternativa hubiese requerido, luego del retiro del implante, la regeneración del sitio del pontico para nivelar el margen mucoso, además del tallado periferico del pilar 2.1 con los riesgos inherentes que tienen este tipo de terapeuticas, cuyas complicaciones, desde el punto de vista biológico son la pérdida de la vitalidad pulpar del diente pilar, seguido por lesiones de caries. Es importante señalar que en la actualidad la implantología presenta ventajas comparativas respecto a la protesis fija plural, como son la conservación del tejido biológico de dientes pilares, lo que implica ser mas conservador y la individualización del tratamiento que evita que el eventual fracaso comprometa a los dientes vecinos.

\section{CONCLUSIÓN}

En el presente trabajo se describen los pasos quirúrgicos y protésicos para resolver un implante con una Clase $\mathrm{IVc}^{(2)}$ en una paciente de 37 años, pasando de un PES/WES ${ }^{(8)}$ inicial de 8 a un valor final de 16 , mejorando la estética del sector anterosuperior.

Este reporte reafirma las indicaciones terapéuticas establecidas recientemente por Zucchelli y cols ${ }^{(2)}$, donde el manejo de tejido blando no ofrece un resultado predecible. Para este tipo de defectos se debe tomar la decisión de extraer el implante y realizar la regeneración de los tejidos cautelando adecuadamente el posicionamiento 3D del implante para obtener un resultado exitoso.

\section{CONFLICTO DE INTERESES}

Los autores declaran no tener conflictos de interés.

\section{Bibliografía}

1. Ferrigno N, Laureti M, Fanali S, Grippaudo G. A long-tern follow- up study of nonsubmerged ITI implants in the treatment of totally edentulous jaws. Part I: Ten-year life table analysis of a prospective multicenter study with 1286 implants. Clin Oral Implants Res. 2002;13:260-73.

2. Zucchelli G, Tavelli L, Stefanini M, Barootchi S, Mazzotti C, Gori G, et al. Classification of facial peri-implant soft tissue dehiscence/ deficiencies at single implant sites in the esthetic zone. J Periodontol. 2019;90:1116-24.

3. Araujo MG, Lindhe J. Dimensional ridge alterations following tooth extraction. An experimental study in the dog. J Clin Periodontol. 2005;32:212-18.

4. Oghli A, Steveling H. Ridge preservation following tooth extraction: A comparison between atraumatic extraction and socket seal surgery. Quintessence Int. 2010;41:605-9.

5. Buser D, Martin W, Belser U. Optimizing esthetics for implant restorations in the anterior maxilla: anatomic and surgical considerations. Int J Oral Maxillofac Implants. 2004;19:43-61.

6. Mazzotti C, Stefanini M, Felice P, Bentivogli V, Mounssif I, Zucchelli G. Soft-tissue dehiscence coverage at peri-implant sites. Periodontol 2000. 2018;77(1):256-72.

7. Bengazi F, Wennstrom J, Lekholm U. Recession of the soft tissue margin at oral implants. A 2-year longitudinal prospective study. Clin Oral Implants Res. 1996;7:303-10

8. Belser U, Grütter L, Vailati F, Bornstein M, Weber H-P, Buser D. Outcome evaluation of early placed maxillary anterior single-tooth implants using objective esthetic criteria: A cross-sectional, retrospective study in 45 patients with a 2- to 4-year follow-up using pink and white esthetic scores. J Periodontol. 2009;80:140-51 9. Elian N, Cho S-C, Froum S, Smith R, Tarnow D. A simplified socket classification and repair technique. Pract Proced Aesthet Dent. 2007;19:99-104.

10. Hürzeler $M$, Weng $D$. A single-incision technique to harvest subepithelial connective tissue grafts from the palate. Int $\mathrm{J}$ Periodontics Restorative Dent. 1999;19:279-87.

11. Wittneben J, Buser D, Belser U, Bragger U. Peri-implant soft tissue conditioning with provisional restorations in the esthetic zone: The dynamic compression technique. Int J Periodont Restor Dent. 2013;33:447-55

12. Zucchelli G, Mazzotti C Mounssif I, Mele M, Stefanini M, Montebugnoli L. A novel surgical-prosthetic approach for soft tissue dehiscence coverage around single implant. Clin Oral Impl Res. 2013;24:957-62.

13. Avila-Ortiz G, Gonzalez-Martin O, Couso-Queiruga E, Wang, HL. The periimplant phenotype. J Periodontol. 2020;91:283-8.

14. Rotundo R, Pagliaro U, Bendinelli E, Esposito M, Buti J. Long-term outcomes of soft tissue augmentation around dental implants on soft and hard tissue stability. A systematic review. Clin Oral Impl Res. 2015;26:123-38.

15. Fürhauser R, Florescu D, Benesch T, Mailath G, Watzek G. Evaluation of soft tissue around single-tooth implant crowns: The pink esthetic score. Clin Oral Implants Res. 2005;16:639-44. 